\title{
NEW MEMBERS OF THE SCORPIUS-CENTAURUS COMPLEX AND AGES OF ITS SUB-REGIONS
}

\author{
INSEOK SONG ${ }^{1}$, B. ZUCKERMAN ${ }^{2}$, AND M. S. BESSELL ${ }^{3}$ \\ ${ }^{1}$ Department of Physics and Astronomy, The University of Georgia, Athens, GA 30602-2451, USA \\ 2 Department of Physics \& Astronomy, University of California, Los Angeles, 475 Portola Plaza, Los Angeles, CA 90095-1547, USA \\ ${ }^{3}$ Research School of Astronomy and Astrophysics, Institute of Advanced Studies, The Australian National University, ACT 2611, Australia \\ Received 2012 March 12; accepted 2012 April 24; published 2012 June 6
}

\begin{abstract}
We have spectroscopically identified $\sim 100 \mathrm{G}-, \mathrm{K}-$, and M-type members of the Scorpius-Centaurus complex. To deduce the age of these young stars we compare their Li $\lambda 6708$ absorption line strengths against those of stars in the TW Hydrae association and $\beta$ Pictoris moving group. These line strengths indicate that Sco-Cen stars are younger than $\beta$ Pic stars whose ages of $\sim 12$ Myr have previously been derived from a kinematic traceback analysis. Our derived age, $\sim 10 \mathrm{Myr}$, for stars in the Lower Centaurus Crux and Upper Centaurus Lupus subgroups of ScoCen is younger than previously published ages based on the moving cluster method and upper main-sequence fitting. The discrepant ages are likely due to an incorrect (or lack of) cross-calibration between model-dependent and model-independent age-dating methods.
\end{abstract}

Key words: open clusters and associations: individual (Scorpius OB2, Lower Centaurus-Crux, Upper Centaurus-Lupus, Upper Scorpius) - stars: activity - stars: kinematics and dynamics - stars: pre-main sequence

Online-only material: color figures

\section{INTRODUCTION}

The Scorpius-Centaurus region (ScoCen) is the nearest (100-200 pc) massive star formation site to Earth. It consists of three subgroups (de Zeeuw et al. 1999): Upper Scorpius (US), Upper Centaurus Lupus (UCL), and Lower Centaurus Crux (LCC). Each of these sub-regions has a different location in the sky plane, different age, and different space motion. Therefore, ScoCen is the best site for studying a sequential star formation or triggered star formation phenomena. Furthermore, ScoCen holds the key to the origin of nearby young stellar groups (Zuckerman \& Song 2004; Fernández et al. 2008), and due to its youth and proximity to Earth, a thorough membership determination can be made down to very low mass.

Using Hipparcos data, de Zeeuw et al. (1999) refined a list of Sco-Cen members containing many B- and A-type stars and relatively few F- and G-type stars. From the number of B- and A-type stars $(N \sim 300)$, several thousand low-mass members were expected to exist. However, mainly due to the vast surface area of ScoCen in the projected sky plane $\left(\sim 2000 \mathrm{deg}^{2}\right)$ and its deep southern declination ( $80 \%$ of the region is below decl. $=-40^{\circ}$ ), this region has been little studied. Compared to a similarly massive but more distant star formation site (e.g., the Orion region), the Sco-Cen complex has been barely investigated. No systematic search for low-mass members of ScoCen has been carried out with the exception of occasional small area pilot surveys (for example, Preibisch et al. 2001).

Mamajek et al. (2002) identified several dozen F- and G-type LCC and UCL members. Using a moving cluster method, they estimated secular parallaxes of new members, then derived ages (UCL 16 Myr and LCC 17 Myr) by plotting them on a Hertzsprung-Russell diagram (HRD) and comparing them with theoretical pre-main-sequence models. Recently, using a larger $(N=138)$ sample of F-type kinematic members from de Zeeuw et al. (1999), Pecaut et al. (2012) re-deduce old ages (16/17 Myr) for the UCL/LCC regions.

Using our $\sim 100$ spectroscopically confirmed G/K/M-type members of LCC/UCL (Table 1), we show that the LCC/UCL age is more consistent with a younger age ( $\sim 10 \mathrm{Myr})$. Because our age determination is anchored in the traceback age for the $\beta$ Pic moving group and because kinematic traceback is the least model-dependent technique for deriving ages of young stars, we expect that an $\sim 10 \mathrm{Myr}$ age for LCC/UCL is most likely to be correct.

Ages of LCC and UCL are important in the interpretation of Spitzer and other data (e.g., Currie et al. 2008). For example, the high fraction $(>35 \%)$ of dusty disks (including several mid-IR excesses) around Sco-Cen F/G stars (Chen et al. 2005) applies to $\sim 10 \mathrm{Myr}$ old stars, rather than stars of nearly twice this age.

\section{NEW MEMBERS}

\subsection{Observations}

As part of an extensive search for young and nearby stars to Earth, $\lambda / \Delta \lambda \sim 4500$ spectra of candidate Sco-Cen members were obtained with the Double Beam Spectrograph (DBS) on the Nasmyth-A focus of the Australian National University's $2.3 \mathrm{~m}$ telescope. For many bright young stars confirmed from DBS spectra, we later obtained echelle spectra to obtain radial velocities. Candidate Sco-Cen members were selected over the Sco-Cen region (Figure 9 of de Zeeuw et al. 1999) from a correlation between X-ray (ROSAT; Voges et al. 1999, 2000) and kinematic catalogs (Hipparcos: Perryman et al. 1997; Tycho-2: Høg et al. 2000; SuperCOSMOS: Hambly et al. 2001). Then, we kept only X-ray bright stars $\left(\log L_{X} / L_{\text {bol }} \gtrsim 10^{-3.5}\right)$ whose space motions are consistent with the nominal value of LCC $\left(U V W=-12,-13,-7 \mathrm{~km} \mathrm{~s}^{-1}\right.$; de Zeeuw et al. 1999, details on space motion calculation are given below). Since most candidate members lack sufficient information to enable direct calculation of their UVW (distances to non-Hipparcos stars and radial velocities for almost all candidate members), we calculated $U V W$ based on photometric distances using an $\sim 10 \mathrm{Myr}$ age and a range of radial velocities $(-50$ to $+50 \mathrm{~km} \mathrm{~s}^{-1}$ ). If an X-ray star can have a Sco-Cen-like $U V W$ for some radial velocities within the above stated range, then we selected it as a candidate Sco-Cen member. Our chosen 
Table 1

Identified Members of Sco-Cen

\begin{tabular}{|c|c|c|c|c|c|c|c|c|c|c|c|c|c|c|}
\hline \multirow[t]{2}{*}{ No. } & \multirow[t]{2}{*}{ Name } & \multirow[t]{2}{*}{ R.A. } & \multirow[t]{2}{*}{ Decl. } & \multirow{2}{*}{$\begin{array}{l}\text { Dist. } \\
\text { (pc) }\end{array}$} & \multirow[t]{2}{*}{$N$} & \multicolumn{2}{|c|}{ Eq. Width } & \multirow{2}{*}{$\begin{array}{c}V \\
(\mathrm{mag})\end{array}$} & \multirow{2}{*}{$\begin{array}{l}V-K \\
(\mathrm{mag})\end{array}$} & \multirow{2}{*}{$\begin{array}{l}B-V \\
(\mathrm{mag})\end{array}$} & \multirow[t]{2}{*}{$f$} & \multirow{2}{*}{$\begin{array}{l}\text { Rad. Vel. } \\
\left(\mathrm{km} \mathrm{s}^{-1}\right)\end{array}$} & \multirow{2}{*}{$\begin{array}{c}(U, V, W) \\
\left(\mathrm{km} \mathrm{s}^{-1}\right)\end{array}$} & \multirow[t]{2}{*}{ Note } \\
\hline & & & & & & $\mathrm{Li}$ & $\mathrm{H} \alpha$ & & & & & & & \\
\hline \multicolumn{15}{|c|}{ Lower Centaurus Crux } \\
\hline 1 & SSS 1132-3019 & $11: 32: 18.38$ & $-30: 19: 51.5$ & 42 & 1 & 500 & -6.7 & $14.20 \mathrm{~d}$ & 5.32 & 1.62 & -3.51 & - & - & phot dist $=55$, TWA 30 \\
\hline 2 & HIP 57524 & $11: 47: 24.58$ & $-49: 53: 02.9$ & 92 & 2 & 190 & 0.6 & 9.07 & 1.56 & 0.65 & -3.29 & $13.4 \pm 1.5$ & $-6.4,-18.7,-5.0$ & phot dist $=86$, TWA 19A \\
\hline 3 & HIP 57524 B & $11: 47: 20.64$ & $-49: 53: 04.2$ & 92 & 1 & 330 & -1.6 & $12.30 \mathrm{~d}$ & 4.13 & 1.52 & -3.29 & $15.3 \pm 3.2$ & $-5.7,-20.4,-4.6$ & phot dist $=63$, TWA 19B \\
\hline 4 & TYC 8631-0128 & $11: 55: 57.75$ & $-52: 54: 00.8$ & 107 & 3 & 360 & 0.1 & 11.00 & 2.61 & 1.06 & -3.50 & $11.5 \pm 1.5$ & $-10.5,-18.5,-4.8$ & \\
\hline 5 & SSS 1159-4510 & 11:59:27.87 & $-45: 10: 19.2$ & 57 & 1 & 420 & -4.6 & $14.54 \mathrm{p}$ & 5.47 & 1.65 & -2.92 & - & - & \\
\hline 6 & SSS 1205-5331 & 12:05:12.66 & $-53: 31: 23.1$ & 102 & 1 & 270 & -1.9 & $13.54 \mathrm{~d}$ & 4.23 & 1.53 & -3.06 & - & - & \\
\hline 7 & HIP 58996 & $12: 05: 47.52$ & $-51: 00: 12.1$ & 110 & 1 & 240 & 0.9 & 8.89 & 1.58 & 0.66 & -3.56 & $16.3 \pm 2.5$ & $-8.6,-24.0,-6.0$ & phot dist $=78.0$ \\
\hline 8 & SSS 1208-5850 & 12:08:20.60 & $-58: 50: 15.1$ & 79 & 1 & 200 & -3.4 & $15.35 \mathrm{~d}$ & 5.53 & 1.67 & -3.08 & - & - & \\
\hline 9 & SSS 1210-4855 & $12: 10: 10.34$ & $-48: 55: 45.9$ & 104 & 1 & 360 & -0.3 & $11.21 \mathrm{p}$ & 2.79 & 1.14 & -3.08 & - & - & \\
\hline 10 & TYC 8636-2515 & $12: 12: 35.79$ & $-55: 20: 27.2$ & 99 & 4 & 300 & 0.2 & 10.48 & 2.36 & 0.95 & -3.22 & $14.7 \pm 1.5$ & $-6.1,-20.9,-6.7$ & \\
\hline 11 & TYC 8978-3494 & $12: 12: 48.93$ & $-62: 30: 31.9$ & 63 & 1 & 350 & 0.4 & 11.77 & 3.81 & 1.38 & -2.52 & - & - & FS 623 \\
\hline 12 & TYC 8978-5124 & 12:13:57.02 & $-62: 55: 12.6$ & 98 & 1 & 310 & -0.2 & 11.40 & 3.01 & 1.28 & -3.03 & - & - & \\
\hline 13 & TYC 8242-1324 & $12: 14: 34.12$ & $-51: 10: 12.4$ & 103 & 1 & 270 & 0.4 & 10.29 & 2.15 & 0.87 & -3.35 & - & - & \\
\hline 14 & TYC 8242-1324B & $12: 14: 31.88$ & $-51: 10: 15.7$ & 84 & 1 & 310 & -0.5 & $13.57 \mathrm{~d}$ & 4.48 & 1.54 & -3.35 & - & - & \\
\hline 15 & HIP 59716 & 12:14:50.76 & $-55: 47: 23.4$ & 97 & 1 & 100 & 1.6 & 8.45 & 1.17 & 0.45 & -3.54 & $13.0 \pm 7.0$ & $-8.1,-19.8,-4.6$ & phot dist $=86$ \\
\hline 16 & HIP 59721 & 12:14:52.35 & $-55: 47: 03.5$ & 97 & 1 & 330 & -0.7 & 9.77 & 2.26 & 0.79 & -3.54 & $17.6 \pm 1.7$ & $-5.8,-23.6,-2.9$ & phot dist $=76$ \\
\hline 17 & TYC 8637-1558 & 12:16:01.20 & $-56: 14: 06.9$ & 69 & 1 & 290 & 0.2 & 11.50 & 3.54 & 1.40 & -2.99 & - & - & broad lines \\
\hline 18 & SSS 1216-5055 & 12:16:17.01 & $-50: 55: 26.3$ & 105 & 1 & 150 & -3.7 & $14.69 \mathrm{~d}$ & 4.83 & 1.57 & -2.14 & - & - & FS 625 \\
\hline 19 & TYC 8986-0497 & $12: 16: 30.10$ & $-67: 11: 47.7$ & 72 & 3 & 430 & -0.2 & 11.10 & 3.25 & 1.39 & -3.19 & $7.0 \pm 5.0$ & $-6.8,-11.5,-5.5$ & \\
\hline 20 & TYC 9231-1185 & $12: 16: 40.31$ & $-70: 07: 36.1$ & 92 & 1 & 325 & 0.0 & 10.73 & 2.64 & 1.07 & -3.49 & - & - & \\
\hline 21 & TYC 8641-2187 & $12: 18: 58.05$ & $-57: 37: 19.1$ & 68 & 4 & 340 & -0.1 & 9.87 & 2.50 & 1.01 & -3.22 & - & - & broad lines \\
\hline 22 & TYC 8983-0098 & $12: 19: 21.68$ & $-64: 54: 10.3$ & 66 & 4 & 340 & 0.2 & 10.12 & 2.72 & 1.11 & -3.21 & $15.0 \pm 1.5$ & $-2.3,-18.3,-5.5$ & \\
\hline 23 & SSS 1219-5018 & $12: 19: 59.38$ & $-50: 18: 38.1$ & 165 & 1 & 260 & -1.0 & $12.89 \mathrm{p}$ & 3.24 & 1.38 & -2.85 & - & - & 2MASS binary?, $\sim 2$ ". $5 \mathrm{NS}$ \\
\hline 24 & TYC 8983-0795 & $12: 20: 54.56$ & $-64: 57: 24.2$ & 98 & 1 & 400 & -0.9 & 10.39 & 2.31 & 0.93 & -3.53 & - & - & \\
\hline 25 & TYC 8983-0564 & $12: 21: 30.84$ & $-64: 03: 52.7$ & 56 & 1 & 380 & -0.8 & 10.83 & 3.42 & 1.40 & -3.33 & - & - & \\
\hline 26 & TYC 8238-1462 & $12: 21: 55.69$ & $-49: 46: 12.4$ & 99 & 2 & 355 & -0.4 & 10.02 & 2.01 & 0.83 & -3.42 & $13.8 \pm 2.4$ & $-7.4,-21.5,-5.3$ & \\
\hline 27 & TYC 8234-2856 & $12: 22: 04.32$ & $-48: 41: 24.8$ & 101 & 1 & 340 & 0.5 & 10.51 & 2.35 & 0.94 & -3.23 & $13.1 \pm 2.3$ & $-5.1,-19.2,-4.0$ & \\
\hline 28 & SSS 1222-5739 & $12: 22: 28.84$ & $-57: 39: 12.2$ & 81 & 1 & 240 & -4.3 & $14.59 \mathrm{~d}$ & 5.09 & 1.59 & -2.81 & - & - & \\
\hline 29 & SSS 1222-6020 & $12: 22: 39.93$ & $-60: 20: 24.4$ & 106 & 1 & 140 & -3.0 & $15.09 \mathrm{~d}$ & 5.04 & 1.58 & -2.76 & - & - & \\
\hline 30 & SSS 1223-5540 & $12: 23: 14.33$ & $-55: 40: 16.1$ & 78 & 1 & 130 & -11.0 & $14.85 \mathrm{~d}$ & 5.27 & 1.60 & -2.47 & - & - & FS 632 \\
\hline 31 & TYC 8983-0854 & $12: 23: 47.54$ & $-64: 02: 54.9$ & 101 & 1 & 375 & -0.7 & 10.79 & 2.55 & 1.03 & -3.41 & - & - & \\
\hline 32 & TYC 8979-1997 & 12:27:16.63 & $-62: 39: 14.2$ & 91 & 1 & 375 & 0.0 & 10.90 & 2.78 & 1.13 & -3.15 & - & - & \\
\hline 33 & TYC 8979-1683 & $12: 28: 25.44$ & $-63: 20: 58.6$ & 73 & 3 & 260 & 0.6 & 9.33 & 2.00 & 0.83 & -2.91 & $13.9 \pm 1.9$ & $-3.0,-17.9,-5.1$ & \\
\hline 34 & TYC 8654-2791 & $12: 33: 33.85$ & $-57: 14: 06.6$ & 101 & 1 & 345 & 0.0 & 10.89 & 2.62 & 1.07 & -3.23 & - & - & \\
\hline 35 & TYC 8992-0605 & $12: 36: 39.02$ & $-63: 44: 43.4$ & 68 & 3 & 410 & 0.3 & 9.88 & 2.51 & 1.01 & -3.38 & - & - & \\
\hline 36 & TYC 8646-0166 & $12: 36: 59.00$ & $-54: 12: 17.9$ & 104 & 1 & 290 & 0.6 & 10.40 & 2.22 & 0.89 & -3.36 & - & - & \\
\hline 37 & TYC 8658-1264 & $12: 38: 35.60$ & $-59: 16: 43.8$ & 123 & 1 & 380 & -0.6 & 11.62 & 2.83 & 1.15 & -3.10 & - & - & \\
\hline 38 & SSS 1244-6902 & $12: 44: 14.57$ & $-69: 02: 35.4$ & 79 & 1 & 520 & -3.3 & $13.34 d$ & 4.43 & 1.54 & -2.64 & - & - & FS 645 \\
\hline 39 & TYC 8992-0420 & $12: 44: 34.85$ & $-63: 31: 46.1$ & 79 & 2 & 390 & -0.9 & 10.79 & 2.91 & 1.20 & -3.05 & - & - & \\
\hline 40 & TYC 8647-0324 & $12: 45: 48.85$ & $-54: 10: 58.3$ & 127 & 1 & 340 & -0.2 & 11.28 & 2.54 & 1.03 & -3.23 & - & - & \\
\hline
\end{tabular}


Table 1

(Continued)

\begin{tabular}{|c|c|c|c|c|c|c|c|c|c|c|c|c|c|c|}
\hline \multirow[t]{2}{*}{ No. } & \multirow[t]{2}{*}{ Name } & \multirow{2}{*}{\multicolumn{2}{|c|}{ (J2000) }} & \multirow{2}{*}{$\begin{array}{l}\text { Dist. } \\
\text { (pc) }\end{array}$} & \multirow[t]{2}{*}{$N$} & \multicolumn{2}{|c|}{ Eq. Width } & \multirow{2}{*}{$\begin{array}{c}V \\
(\mathrm{mag})\end{array}$} & \multirow{2}{*}{$\begin{array}{l}V-K \\
(\mathrm{mag})\end{array}$} & \multirow{2}{*}{$\begin{array}{l}B-V \\
(\mathrm{mag})\end{array}$} & \multirow[t]{2}{*}{$f$} & \multirow{2}{*}{$\begin{array}{l}\text { Rad. Vel. } \\
\left(\mathrm{km} \mathrm{s}^{-1}\right)\end{array}$} & \multirow{2}{*}{$\begin{array}{c}(U, V, W) \\
\left(\mathrm{km} \mathrm{s}^{-1}\right)\end{array}$} & \multirow[t]{2}{*}{ Note } \\
\hline & & & & & & $\mathrm{Li}$ & $\mathrm{H} \alpha$ & & & & & & & \\
\hline 41 & TYC 9228-1355 & $12: 47: 21.99$ & $-68: 08: 40.0$ & 86 & 1 & 425 & -0.5 & 10.88 & 2.85 & 1.16 & -3.50 & - & - & \\
\hline 42 & SSS 1247-5050 & $12: 47: 35.99$ & $-50: 50: 51.9$ & 107 & 1 & 140 & -4.7 & $14.98 \mathrm{~d}$ & 4.97 & 1.58 & -2.98 & - & - & \\
\hline 43 & TYC 8651-0442 & $12: 47: 48.27$ & $-54: 31: 30.6$ & 75 & 1 & 460 & -2.2 & 11.47 & 3.42 & 1.40 & -3.06 & - & - & \\
\hline 44 & TYC 7783-1908 & $12: 48: 07.82$ & $-44: 39: 16.6$ & 76 & 1 & 260 & 0.0 & 9.73 & 2.22 & 0.89 & -3.11 & - & - & \\
\hline 45 & TYC 8257-1545 & $12: 50: 51.44$ & $-51: 56: 35.4$ & 109 & 1 & 450 & -2.1 & 11.68 & 3.05 & 1.30 & -3.35 & - & - & 2MASS binary? $\sim 3^{\prime \prime} \mathrm{EW}$ \\
\hline 46 & SSS 1251-5253 & $12: 51: 05.57$ & $-52: 53: 12.1$ & 102 & 1 & 395 & -1.1 & $12.39 \mathrm{~d}$ & 3.57 & 1.41 & -2.87 & - & - & FS 650 \\
\hline 47 & SSS 1251-5630 & $12: 51: 12.46$ & $-56: 30: 46.8$ & 70 & 1 & 460 & -2.1 & $13.24 d$ & 4.51 & 1.54 & -3.17 & - & - & \\
\hline 48 & SSS 1252-5615 & $12: 52: 00.60$ & $-56: 15: 57.7$ & 93 & 1 & 340 & -4.7 & $14.52 \mathrm{~d}$ & 4.87 & 1.57 & -2.71 & - & - & \\
\hline 49 & SSS 1252-5553 & $12: 52: 14.72$ & $-55: 53: 37.2$ & 109 & 1 & 360 & -1.7 & $12.54 \mathrm{~d}$ & 3.57 & 1.41 & -3.12 & - & - & FS 652 \\
\hline 50 & SSS 1255-5355 & $12: 55: 55.95$ & $-53: 55: 31.1$ & 99 & 1 & 315 & -4.4 & $14.50 \mathrm{~d}$ & 4.80 & 1.56 & -2.90 & - & - & \\
\hline 51 & TYC 9245-0535 & $12: 56: 08.35$ & $-69: 26: 53.9$ & 68 & 1 & 430 & -1.9 & 11.63 & 3.64 & 1.41 & -2.66 & - & - & FS 655 \\
\hline 52 & TYC 8989-0583 & $12: 56: 09.46$ & $-61: 27: 25.3$ & 68 & 3 & 260 & -0.1 & 9.45 & 2.19 & 0.88 & -2.93 & $10.5 \pm 3.0$ & $-8.3,-18.3,-4.1$ & \\
\hline 53 & TYC 9245-0617 & $12: 58: 25.65$ & $-70: 28: 49.0$ & 75 & 3 & 350 & 0.0 & 9.92 & 2.38 & 0.95 & -3.32 & $11.1 \pm 1.5$ & $-7.3,-17.1,-7.5$ & \\
\hline 54 & SSS 1259-6808 & 12:59:35.74 & $-68: 08: 01.0$ & 59 & 1 & 540 & -6.3 & $14.56 \mathrm{~d}$ & 5.44 & 1.65 & -1.90 & - & - & FS 658 \\
\hline 55 & TYC 8648-0446 & 13:01:50.70 & $-53: 04: 58.3$ & 136 & 1 & 290 & 0.2 & 11.09 & 2.30 & 0.92 & -3.28 & - & - & \\
\hline 56 & TYC 8993-0409 & 13:02:47.06 & $-62: 13: 58.9$ & 88 & 1 & 315 & 0.8 & 10.18 & 2.33 & 0.93 & -3.25 & - & - & 2MASS binary $\sim 9^{\prime \prime} \mathrm{NE}$ \\
\hline 57 & TYC 9242-0290 & 13:14:01.15 & $-68: 46: 38.5$ & 106 & 1 & 360 & -0.4 & 11.27 & 2.81 & 1.14 & -3.27 & - & - & SB2? \\
\hline 58 & TYC 8259-0689 & $13: 14: 23.86$ & $-50: 54: 01.8$ & 99 & 1 & 250 & 0.5 & 10.40 & 2.30 & 0.92 & -2.97 & - & - & \\
\hline 59 & TYC 8674-2317 & $13: 21: 20.30$ & $-59: 03: 44.0$ & 73 & 2 & 430 & -1.2 & 10.82 & 3.04 & 1.30 & -3.50 & $15.5 \pm 1.8$ & $-1.3,-20.8,-4.4$ & \\
\hline 60 & HIP 65423 & $13: 24: 35.15$ & $-55: 57: 24.0$ & 124 & 1 & 220 & 0.9 & 9.59 & 1.51 & 0.63 & -3.51 & $8.1 \pm 2.1$ & $-8.9,-17.9,-4.3$ & phot dist $=106.2$ \\
\hline 61 & TYC 8256-1840 & $13: 27: 05.98$ & $-48: 56: 17.9$ & 77 & 1 & 350 & -0.6 & 10.69 & 2.88 & 1.18 & -3.12 & - & - & \\
\hline 62 & TYC 7796-2110 & 13:34:31.92 & $-42: 09: 30.5$ & 93 & 1 & 315 & -0.9 & 10.70 & 2.61 & 1.06 & -3.10 & - & - & \\
\hline 63 & TYC 7796-1788 & 13:37:57.32 & $-41: 34: 41.7$ & 91 & 1 & 275 & 0.6 & 10.08 & 2.20 & 0.88 & -3.20 & - & - & \\
\hline 64 & TYC 7800-0858 & 13:38:05.99 & $-43: 44: 56.3$ & 114 & 1 & 310 & 0.0 & 11.14 & 2.60 & 1.06 & -3.49 & - & - & \\
\hline 65 & TYC 7796-0286 & 13:38:49.37 & $-42: 37: 23.4$ & 138 & 1 & 320 & 0.1 & 11.36 & 2.47 & 0.99 & -3.64 & - & - & \\
\hline 66 & TYC 8261-1690 & 13:40:25.56 & $-46: 33: 51.3$ & 102 & 2 & 340 & -1.5 & 11.38 & 2.94 & 1.23 & -2.79 & $10.1 \pm 1.9$ & $-2.1,-17.0,-2.7$ & \\
\hline 67 & TYC 8266-2914 & 13:44:24.45 & $-47: 06: 33.9$ & 93 & 4 & 310 & 0.5 & 10.50 & 2.47 & 0.99 & -3.47 & - & - & \\
\hline 68 & TYC 9012-1005 & 13:44:42.84 & $-63: 47: 49.2$ & 70 & 4 & 370 & -0.4 & 10.88 & 3.14 & 1.37 & -3.16 & $18.0 \pm 1.0$ & $-1.2,-24.0,-4.1$ & SB? \\
\hline 69 & TYC 8274-0030 & 13:45:56.02 & $-52: 22: 25.3$ & 114 & 1 & 340 & -0.1 & 11.34 & 2.75 & 1.12 & -2.98 & - & - & \\
\hline 70 & TYC 8267-2879 & 13:54:42.13 & $-48: 20: 57.6$ & 129 & 1 & 260 & 0.0 & 11.07 & 2.37 & 0.95 & -3.24 & - & - & \\
\hline 71 & TYC 8271-0864 & 13:56:34.69 & $-49: 07: 14.5$ & 136 & 4 & 310 & -0.2 & 11.14 & 2.33 & 0.93 & -3.10 & $5.6 \pm 1.7$ & $-11.8,-20.7,-4.3$ & \\
\hline \multicolumn{15}{|c|}{ Upper Centaurus Lupus } \\
\hline 72 & TYC 7818-0504 & $14: 30: 13.56$ & $-43: 50: 09.7$ & 72 & 1 & 340 & -0.7 & 10.46 & 2.82 & 1.15 & -2.80 & - & - & \\
\hline 73 & SSS $1450-3458$ & 14:50:34.04 & $-34: 58: 56.1$ & 116 & 2 & 370 & -0.7 & $12.10 \mathrm{p}$ & 3.22 & 1.38 & -3.12 & $1.3 \pm 1.6$ & - & No proper motion data \\
\hline 74 & TYC 7325-0465 & $15: 24: 32.37$ & $-36: 52: 02.5$ & 154 & 1 & 340 & 0.2 & 10.87 & 1.94 & 0.81 & -3.41 & $4.0 \pm 1.5$ & $-5.0,-23.3,-4.6$ & \\
\hline 75 & SSS 1533-3917 & $15: 33: 40.48$ & $-39: 17: 47.7$ & 62 & 2 & 280 & -7.9 & $14.22 \mathrm{u}$ & 5.20 & 1.60 & -3.51 & $-2.0 \pm 5.0$ & $-12.9,-34.7,-23.0$ & \\
\hline 76 & SSS 1539-3451 & $15: 39: 46.38$ & $-34: 51: 02.6$ & 83 & 1 & 385 & -0.2 & $12.89 \mathrm{~d}$ & 4.12 & 1.52 & -3.02 & - & - & \\
\hline 77 & HIP 77199 & $15: 45: 47.65$ & $-30: 20: 54.9$ & 40 & 3 & 400 & -0.8 & 9.37 & 2.91 & 1.20 & -3.03 & $-5.7 \pm 1.5$ & $-10.1,-20.2,-7.4$ & phot dist=17.4 (binary?) \\
\hline 78 & TYC 6782-0900 & $15: 47: 07.49$ & $-25: 19: 46.4$ & 92 & 1 & 450 & -0.4 & 11.00 & 2.83 & 1.15 & -3.04 & $-4.5 \pm 1.5$ & $-8.2,-22.6,-5.8$ & \\
\hline 79 & TYC 7328-1706 & 15:49:02.72 & $-31: 02: 53.6$ & 121 & 1 & 350 & -0.1 & 10.85 & 2.30 & 0.92 & -3.28 & $-9.9 \pm 3.5$ & $-13.9,-14.8,-4.9$ & \\
\hline
\end{tabular}


Table 1

(Continued)

\begin{tabular}{|c|c|c|c|c|c|c|c|c|c|c|c|c|c|c|}
\hline \multirow[t]{2}{*}{ No. } & \multirow[t]{2}{*}{ Name } & R.A. & Decl. & \multirow{2}{*}{$\begin{array}{l}\text { Dist. } \\
\text { (pc) }\end{array}$} & \multirow[t]{2}{*}{$N$} & \multicolumn{2}{|c|}{ Eq. Width } & \multirow{2}{*}{$\begin{array}{c}V \\
(\mathrm{mag})\end{array}$} & \multirow{2}{*}{$\begin{array}{l}V-K \\
(\mathrm{mag})\end{array}$} & \multirow{2}{*}{$\begin{array}{l}B-V \\
(\mathrm{mag})\end{array}$} & \multirow[t]{2}{*}{$f$} & \multirow{2}{*}{$\begin{array}{l}\text { Rad. Vel. } \\
\left(\mathrm{km} \mathrm{s}^{-1}\right)\end{array}$} & \multirow{2}{*}{$\begin{array}{l}(U, V, W) \\
\left(\mathrm{km} \mathrm{s}^{-1}\right)\end{array}$} & \multirow[t]{2}{*}{ Note } \\
\hline & & \multicolumn{2}{|c|}{$(\mathrm{J} 2000)$} & & & $\mathrm{Li}$ & $\mathrm{H} \alpha$ & & & & & & & \\
\hline 80 & TYC 7846-1538 & $15: 53: 27.32$ & $-42: 16: 00.2$ & 51 & 3 & 190 & 0.8 & 7.86 & 1.52 & 0.64 & -3.45 & $-0.2 \pm 3.5$ & $-6.9,-16.6,-5.4$ & \\
\hline 81 & TYC 7846-0833 & $15: 56: 44.01$ & $-42: 42: 29.9$ & 78 & 1 & 495 & -1.9 & 11.88 & 3.61 & 1.41 & -3.10 & - & - & \\
\hline 82 & HIP 78345 & $15: 59: 49.53$ & $-36: 28: 27.5$ & 65 & 1 & 425 & -0.3 & 11.00 & 2.97 & 1.25 & -3.20 & $-0.3 \pm 1.2$ & $-3.4,-13.4,-7.7$ & phot dist $=79.0$ \\
\hline 83 & SSS $1603-4018$ & 16:03:05.46 & $-40: 18: 25.8$ & - & 1 & 310 & -60.5 &.-- & - & - & - & - & - & EX Lup \\
\hline 84 & SSS 1606-2036 & $16: 06: 31.70$ & $-20: 36: 23.2$ & 70 & 1 & 450 & -1.3 & $13.47 \mathrm{~d}$ & 4.64 & 1.55 & -3.04 & - & - & FS 810 \\
\hline 85 & TYC 7349-2447 & $16: 35: 22.41$ & $-33: 28: 52.2$ & 72 & 1 & 415 & -0.8 & 11.66 & 3.58 & 1.41 & -2.94 & - & - & \\
\hline 86 & SSS 1639-3920 & $16: 39: 47.32$ & $-39: 20: 40.5$ & 99 & 1 & 440 & -2.0 & $14.08 \mathrm{~d}$ & 4.56 & 1.55 & -2.39 & - & - & FS 844 \\
\hline 87 & SSS 1652-3359 & $16: 52: 10.87$ & $-33: 59: 33.3$ & 86 & 2 & 325 & -1.1 & $11.98 \mathrm{~d}$ & 3.56 & 1.40 & -3.08 & - & - & \\
\hline \multicolumn{15}{|c|}{ Upper Scorpius and Young Stars in the Vicinity } \\
\hline 88 & TYC 6801-0186 & 16:14:59.19 & $-27: 50: 22.7$ & 130 & 1 & 355 & 0.2 & 10.98 & 2.29 & 0.92 & -3.33 & $-2.0 \pm 1.5$ & $-3.6,-16.6,-6.1$ & \\
\hline 89 & TYC 6798-0544 & $16: 25: 19.26$ & $-24: 26: 52.5$ & 87 & 1 & 400 & 1.1 & 10.06 & 2.26 & 0.91 & -3.50 & $-2.4 \pm 1.5$ & $-3.6,-13.7,-1.9$ & \\
\hline 90 & TYC 7344-0788 & $16: 26: 57.65$ & $-30: 32: 27.7$ & 98 & 2 & 440 & -0.5 & 11.68 & 3.19 & 1.38 & -3.09 & $-4.2 \pm 1.6$ & - & \\
\hline 91 & TYC 7344-0788B & $16: 26: 57.00$ & $-30: 32: 23.3$ & 94 & 2 & 425 & -1.0 & $12.46 \mathrm{~d}$ & 3.72 & 1.43 & -3.09 & - & - & \\
\hline 92 & TYC 6816-0234 & $17: 13: 32.84$ & $-26: 02: 06.9$ & 101 & 1 & 350 & 0.6 & 10.14 & 2.08 & 0.85 & -3.17 & $-7.0 \pm 5.0$ & $-6.9,-19.5,-4.2$ & \\
\hline 93 & TYC 6820-0223 & $17: 15: 03.62$ & $-27: 49: 39.4$ & 59 & 1 & 580 & -2.3 & 10.56 & 3.18 & 1.38 & -3.15 & $-1.1 \pm 1.8$ & $-0.9,-10.6,-6.8$ & \\
\hline 94 & HIP 84642 & $17: 18: 14.71$ & $-60: 27: 26.7$ & 59 & 3 & 185 & 0.6 & 9.51 & 1.98 & 0.82 & -3.42 & $0.6 \pm 1.2$ & $-14.3,-26.4,-1.0^{\ddagger}$ & phot dist $=65.3$ \\
\hline 95 & SSS $1719-4615$ & 17:19:42.09 & $-46: 15: 26.5$ & 35 & 2 & 520 & -10.2 & $12.93 p$ & 5.17 & 1.59 & -3.25 & - & - & Wack3672, Flare star, \\
\hline 96 & SSS 1724-3914 & $17: 24: 53.51$ & $-39: 14: 43.8$ & 128 & 2 & 275 & 0.0 & $11.88 \mathrm{p}$ & 2.94 & 1.23 & -3.46 & $-3.2 \pm 1.9$ & $-12.6,-42.1,-32.4$ & \\
\hline 97 & TYC 8728-2262 & $17: 29: 55.08$ & $-54: 15: 48.1$ & 72 & 2 & 310 & 0.2 & 9.54 & 2.18 & 0.88 & -3.21 & $-0.5 \pm 3.7$ & $-9.8,-17.2,-8.9$ & \\
\hline 98 & TYC 5672-0216 & $17: 37: 46.48$ & $-13: 14: 45.6$ & 45 & 3 & 260 & -0.8 & 10.11 & 3.27 & 1.39 & -2.56 & - & - & FS 903 \\
\hline 99 & HIP 86598 & $17: 41: 49.04$ & $-50: 43: 27.5$ & 72 & 2 & 190 & 0.9 & 8.33 & 1.34 & 0.51 & -3.65 & $1.7 \pm 1.7$ & $-7.0,-19.4,-10.5$ & phot dist $=71.3$ \\
\hline 100 & TYC 8742-2065 & $17: 48: 33.74$ & $-53: 06: 42.9$ & 55 & 3 & 260 & 0.4 & 8.99 & 2.21 & 0.89 & -3.15 & $-0.2 \pm 1.5$ & $-6.2,-12.0,-5.9$ & \\
\hline 101 & SSS 1751-4854 & $17: 51: 34.16$ & $-48: 54: 55.4$ & 54 & 2 & 290 & -3.8 & $13.14 \mathrm{~d}$ & 4.76 & 1.56 & -2.93 & - & - & \\
\hline 102 & SSS 1814-3246 & 18:14:22.09 & $-32: 46: 10.8$ & 71 & 1 & 125 & -1.6 & $12.79 \mathrm{p}$ & 4.25 & 1.53 & -2.65 & - & - & \\
\hline 103 & SSS $1818-3710$ & $18: 18: 35.44$ & $-37: 10: 11.5$ & 60 & 2 & 330 & -7.1 & $14.65 \mathrm{~d}$ & 5.70 & 1.71 & -3.02 & - & - & \\
\hline 104 & TYC 7408-0054 & $18: 50: 44.47$ & $-31: 47: 46.8$ & 50 & 2 & 425 & -1.6 & 11.31 & 3.85 & 1.47 & -3.09 & $-3.0 \pm 6.0$ & $-4.1,-16.3,-8.6$ & \\
\hline
\end{tabular}

Notes.

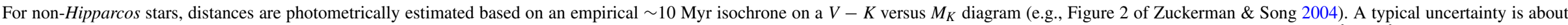

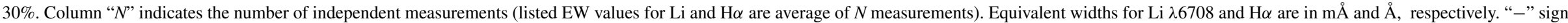

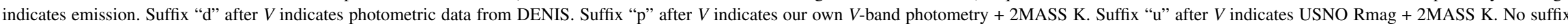

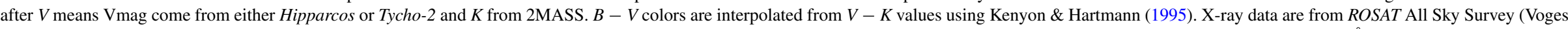

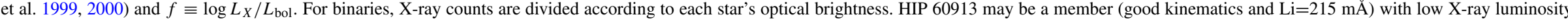
$\left(\log L_{X} / L_{\mathrm{bol}}=-4.34\right)$. HIP 76063 may be a member (A-type star located on zero-age main sequence; Zuckerman \& Song 2004).

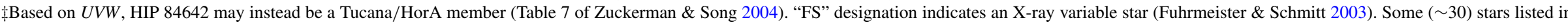
the above table were previously identified by Mamajek et al. (2002). 


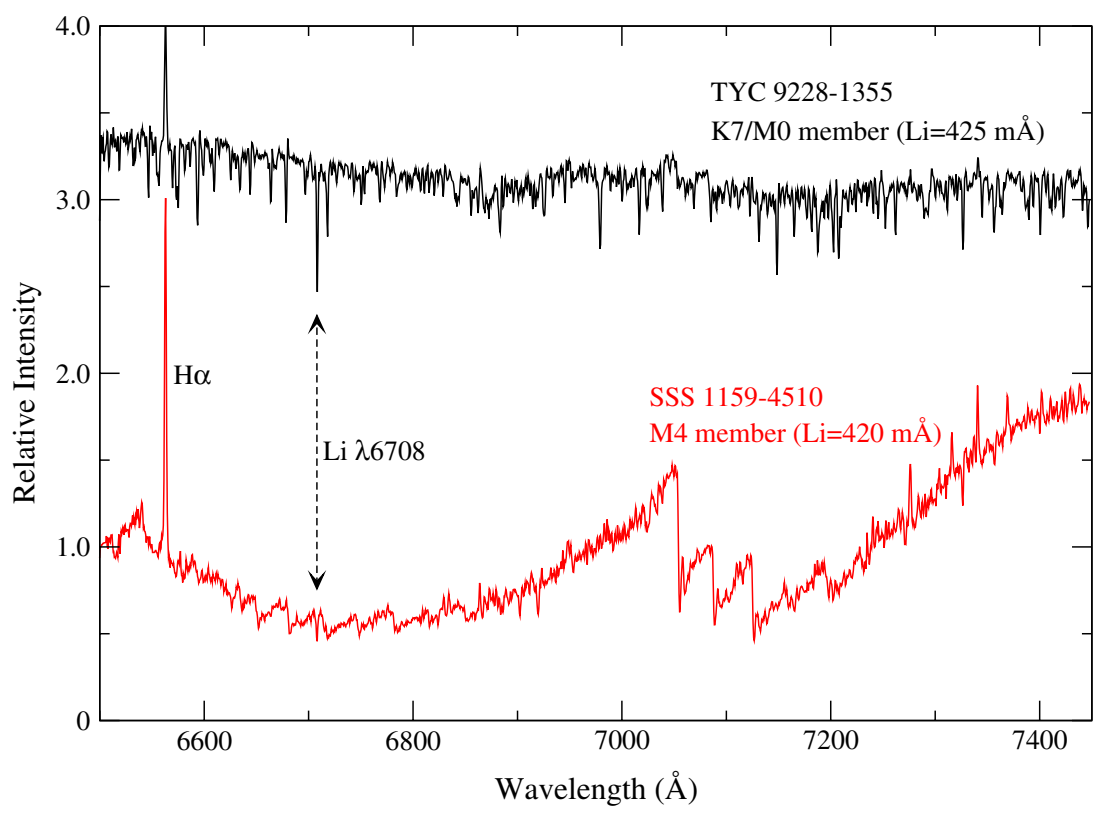

Figure 1. Representative spectra of newly identified Sco-Cen members.

(A color version of this figure is available in the online journal.)

radial velocity range is large enough to cover nearly all ScoCen members because a typical speed $\left(\equiv \sqrt{U^{2}+V^{2}+W^{2}}\right)$ of Sco-Cen stars is less than $30 \mathrm{~km} \mathrm{~s}^{-1}$, requiring that their radial velocities be smaller than this total speed. The use of young age in the photometric distance estimate does not affect the candidate selection much because of our rather generous $U V W$ range ( $\pm 5 \mathrm{~km} \mathrm{~s}^{-1}$ in each component).

All spectra were reduced with IRAF following a standard procedure (bad pixel and cosmic ray removal, flat fielding, source extraction, telluric correction, etc.). Typical spectra have $\sim 5000$ counts pixel $^{-1}$ in the vicinity of $6700 \AA$. Equivalent widths (EWs) of $\mathrm{Li}$ I $\lambda 6708$ and $\mathrm{H} \alpha$ together with their $V-K$ colors and X-ray information are listed in Table 1 . We used $V-K$ colors (see footnotes of Table 1 for sources of $V$ and $K$ magnitudes) as spectral type proxies because $V-K$ separates $\mathrm{K}$ - and M-type subclasses nicely and the long color baseline is less susceptible to measurement errors and time variabilities compared to other broadband colors (e.g., $B-V$ ). Two typical spectra are displayed in Figure 1.

\subsection{Refinement of LCC and UCL Ages}

To estimate ages of LCC and UCL from our spectra, in Figure 2 we compare their $\mathrm{Li} \lambda 6708$ absorption strengths against those of other young stellar groups with well-known ages on an EW(Li) versus $V-K$ plot. Because Table 1 contains US stars that are thought to be $\sim 5 \mathrm{Myr}$ old, we plot LCC/UCL stars and US stars with different symbols in Figure 2. Ages of the TW Hydrae Association ( $~ 8 \mathrm{Myr}$ ) and the $\beta$ Pictoris Moving Group $(\sim 12 \mathrm{Myr})$ are well established and calibrated against contemporary theoretical pre-main-sequence evolutionary models by plotting their members on a color-magnitude diagram (CMD) along with theoretical models. An essentially modelindependent age of the $\beta$ Pictoris moving group was obtained by tracing positions of its members backward in time (Ortega et al. 2002; Song et al. 2003). Members of this unbound stellar group should have been confined in the smallest volume at its birth; a kinematic age of $\sim 12$ Myr obtained from this method agrees well with current stellar evolution models. Similarly, a kinematic age of TWA is estimated to be $8.3 \pm 0.8 \mathrm{Myr}$ (de la Reza et al. 2006).

As is evident in Figure 2, overall lithium absorption strengths of LCC/UCL stars fall between those of the TWA and the $\beta$ Pictoris moving group. Therefore, a likely age of LCC/UCL is $\sim 10$ Myr. Reddening toward the LCC/UCL region $\left(A_{J}=0.00-0.35\right.$ mag; Mamajek et al. 2002) does not change the relative ordering of $\mathrm{Li} \lambda 6708$ strength distribution among TWA, LCC/UCL, and $\beta$ Pictoris Moving Group members. In fact, dereddening will make most LCC/UCL stars appear younger (i.e., moving LCC/UCL stars leftward in Figure 2) because reddening toward the TWA and the $\beta$ Pictoris Moving Group is almost negligible. For this reason, we do not consider the effect of reddening in this paper.

Current theoretical stellar evolutionary models (e.g., Baraffe et al. 1998) predict near complete depletion of lithium (down to the $1 \%-2 \%$ level) among M1/2 stars $(V-K \sim 4.0)$ within $16 \mathrm{Myr}$, but we do not see such depletion of lithium among early M-type LCC/UCL stars (i.e., $V-K \sim 4.0$ ) in Figure 2. In addition, lithium depletion rates predicted in current evolutionary models appear to be slower than what is observed (e.g., Song et al. 2002) which further strengthens the preceding statement. As demonstrated in Figure 2, LCC/UCL members are younger than $\beta$ Pictoris moving group members. Therefore, the LCC/UCL cannot be as old as $16 \mathrm{Myr}$.

Due to our target selection criterion based on ROSAT allsky X-ray detection, our Table 1 LCC stars are systematically closer than stars considered by Mamajek et al. (2002). Therefore, it is conceivable that our LCC/UCL stars (close to Earth) are $\sim 10$ Myr old while more distant LCC/UCL stars surveyed by Mamajek et al. (2002) could be 17/16 Myr old. Supporting this conjecture, although based on small number statistics, Lawson \& Crause (2005) photometrically measure the median rotational period of TWA $1-13$ (4.7 days) to be longer than the median value of TWA 14-19 (0.7 days) which they interpret as an age difference between these two groups, TWA 1-13 being younger than TWA 14-19. To investigate the possibility of age dependence on distance for our low-mass stars, we divided the LCC/UCL stars of Table 1 into two groups (distance $\leqq 95$ pc 


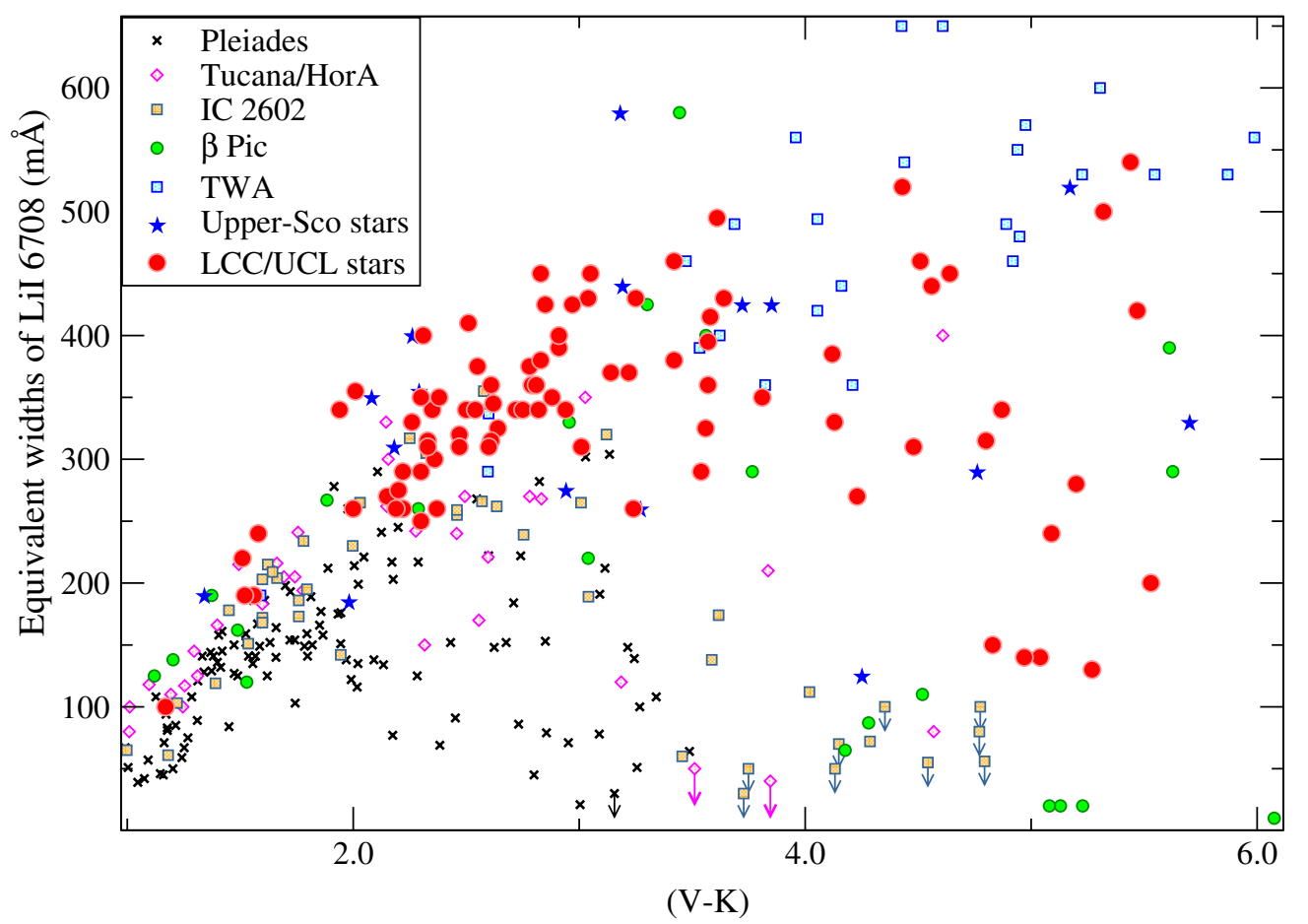

Figure 2. Li $\lambda 6708$ equivalent widths of LCC and UCL stars. Tucana/HorA and IC 2602 stars are generally considered to be $\sim 30 \mathrm{Myr}$ old.

(A color version of this figure is available in the online journal.)

$[N=46]$ and distance $>95 \mathrm{pc}[N=41])$ and compared their Li absorption strengths. Stars in these two bins show almost identical Li $\lambda 6708$ absorption strength distribution, hence we believe that the whole LCC/UCL group is $\sim 10$ Myr old. Furthermore, TWA 14-19 all show very strong Li $\lambda 6708$ absorption strengths that are consistent with LCC stars in Table 1. Nonetheless, whether there is a radial age spread toward the direction of LCC/UCL or not, from Figure 2 alone, $\mathrm{G} / \mathrm{K} / \mathrm{M}$-type members of LCC/UCL are younger than $\sim 12 \mathrm{Myr}$ old. Also, since the original discovery of TWA, many more members have been identified to date. These newly discovered members are generally more distant than original TWA members. As a result, the apparent clear distinction in distance between original TWA members and LCC members is disappearing. The current set of age-dating methods cannot readily discern $\sim 8$ Myr old stars from $\sim 10$ Myr ones. Based on several common characteristics (similar ages, similar positions on the projected sky plane, similar space motions) and the weakening gap in distance between TWA and LCC, we believe that TWA is likely a near edge of a larger population of stars (i.e., LCC). As more sensitive data become available in future, namely next generation parallax measurements, one may find that the distribution of $\sim 10$ Myr old stars extends from TWA to all the way to LCC.

We note that we base our age estimate on Li-strong stars in the relative age dating of TWA, LCC/UCL, and $\beta$ Pictoris moving group members. One might therefore question whether the possible existence and non-inclusion of Li-weak true members might vitiate the validity of such a comparison. Currently, in the absence of accurate trigonometric parallax, there is no effective way to identify Li-depleted members of young dispersed moving groups. This means that the same possible bias introduced by including only Li-strong members exists equally in the TWA, LCC/UCL, and the $\beta$ Pictoris Moving Group. Therefore, comparing the upper envelopes of Li $\lambda 6708$ strength distributions among young stellar groups should be a perfectly valid method of relative age dating.

\subsection{Comparison between HRD and CMD/Li Ages}

Among several commonly used age-dating methodsposition on a CMD or HRD, stellar rotation, Li $\lambda 6708$ absorption strength, $\mathrm{X}$-ray brightness, $\mathrm{H} \alpha$ emission strength, Galactic space motion, Ca II HK index, IR excess emission-the CMD, $\mathrm{HRD}$, and Li methods can provide quantitative age estimates for stars in the 5-30 Myr age range. We already demonstrate the Li age in the previous section. Using the CMD/HRD age-dating method requires a precise distance to a star, and eight stars in Table 1 have measured trigonometric parallaxes from Hipparcos (van Leeuwen 2007). Using the transformation scheme from colors to effective temperatures and bolometric correction values from Mamajek et al. (2002), we plot four LCC/UCL Hipparcos stars on an HRD (Figure 3, left panel). Hipparcos stars from Table 1 sit on the theoretical $20 \mathrm{Myr}$ isochrone from Siess et al. (2000), at first glance apparently in support of an age of 20 Myr as deduced by Mamajek et al. (2002). However, as may be seen, various F-type $\beta$ Pictoris moving group members sit on the $\sim 30 \mathrm{Myr}$ isochrone which is inconsistent with the age of the group $(\sim 12 \mathrm{Myr})$. It implies an age calibration problem between these two age-dating methods similar to the case of inconsistent ages from Ca II HK and Li ages (Song et al. 2004).

In Figure 3, bottom panel, we plot eight Hipparcos Table 1 stars on a $V-K$ versus $M_{K}$ diagram (i.e., model-independent) along with an empirical $10 \mathrm{Myr}$ isochrone from Zuckerman et al. (2004) and several dozen $~ 30$ Myr old stars from TucanaHorologium Association and IC 2602. A useful comparison would be plotting $\sim 20 \mathrm{Myr}$ old F/G-type stars but there are no such suitable stars with reliably determined ages in the solar neighborhood. As shown in the CMD, all eight Hipparcos LCC/UCL stars are located on or above the $10 \mathrm{Myr}$ isochrone as defined by $\eta$ Cha, TWA, and the $\beta$ Pictoris moving group 


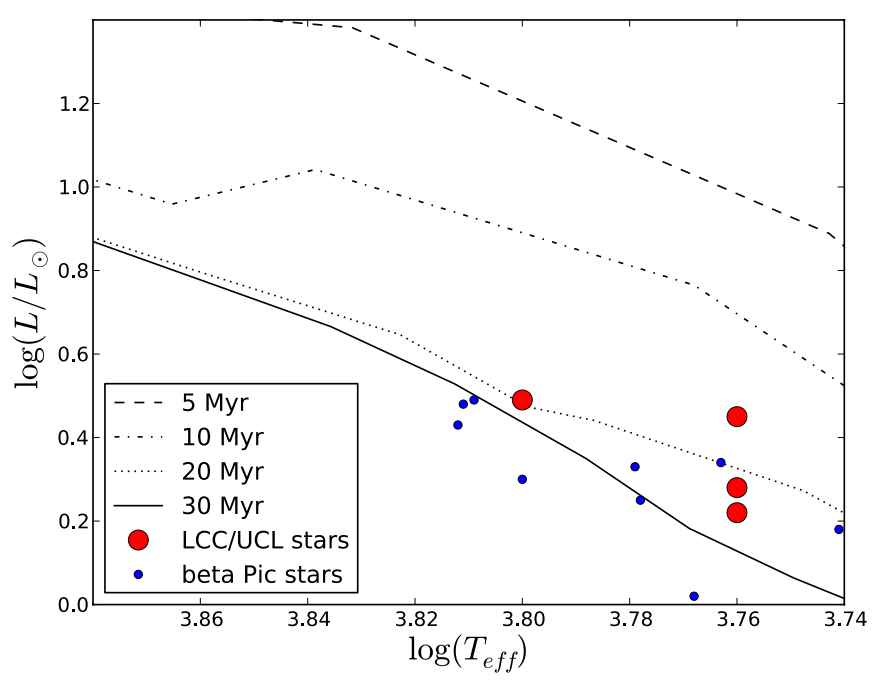

(a) HRD

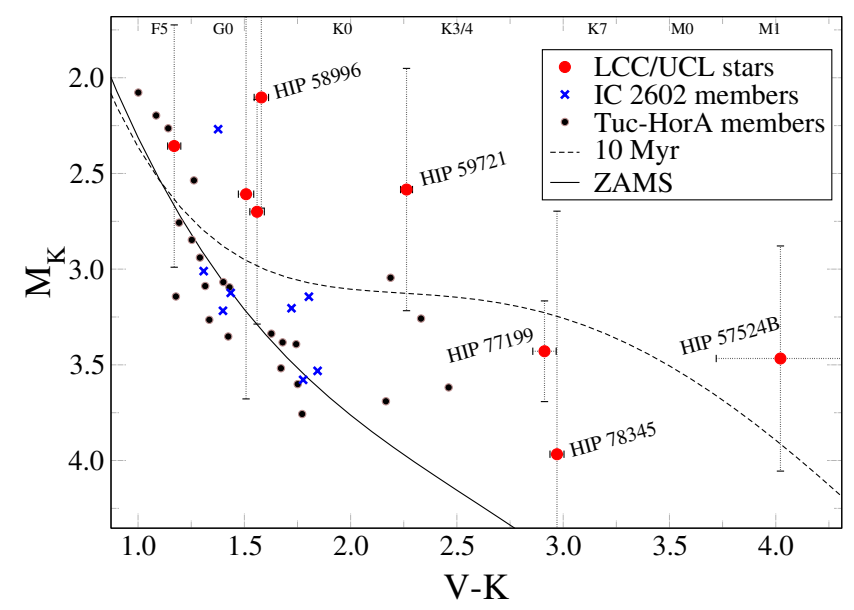

(b) CMD

Figure 3. Top: LCC/UCL stars plotted on a HRD along with theoretical isochrones from Siess et al. (2000). Several F-type $\beta$ Pictoris moving group (BPMG) members are located around the $\sim 30$ Myr theoretical isochrone while the trusted age of the BPMG is based on various techniques that all point to an age of $\sim 12 \mathrm{Myr}$. This implies a significant systematic discrepancy between HRD ages and ages obtained by other methods. Bottom: same LCC/UCL Hipparcos stars plotted on a CMD which does not involve any theoretical models. When compared to empirical $\sim 10 \mathrm{Myr}$ isochrones (from $\eta$ Cha, TWA, and BPMG members) and other slightly older ( $\sim 30 \mathrm{Myr})$ stars from Tucana-HorA and IC 2602, LCC/UCL stars appear to be $\sim 10 \mathrm{Myr}$ in the observational domain. Distances are obtained from the reanalyzed Hipparcos data (van Leeuwen 2007). The distance to HIP 59721 is adapted from its comoving companion (HIP 59716; $\left.\pi=10.36 \pm 1.31 \mathrm{mas} \mathrm{yr}^{-1}\right)$ due to the large error $\left(\pi=7.56 \pm 5.84 \mathrm{mas} \mathrm{yr}^{-1}\right)$ for HIP 59721. For IC 2602, $(m-M)_{0}=5.95$ was used following Stauffer et al. (1997).

(A color version of this figure is available in the online journal.)

(see Zuckerman et al. 2004 for more details on the $10 \mathrm{Myr}$ empirical isochrone). This is a corroborant demonstration that LCC/UCL stars are as young as stars in $\eta$ Cha, TWA, and $\beta$ Pictoris moving group, and the claimed older age of LCC/UCL is likely due either to a lack or incorrect calibration of HRD ages against empirical ages. Likewise, the relatively old age for the Upper-Sco region recently deduced by Pecaut et al. (2012) requires additional scrutiny because it is based on the same HRD age-dating method.

\section{SUMMARY}

We spectroscopically identified $\sim 100 \mathrm{G} / \mathrm{K} / \mathrm{M}$-type Sco-Cen members, mostly LCC and UCL members, that show strong Li $\lambda 6708$ absorption and/or $\mathrm{H} \alpha$ emission features. Comparison of Li absorption strengths against those of other young stellar groups on a $V-K$ versus lithium strength diagram indicates that the age of LCC/UCL is $\sim 10$ Myr. Specifically, LCC/ UCL stars must be younger than stars in the $\beta$ Pictoris moving group whose age of $12 \mathrm{Myr}$ has been derived previously from kinematic traceback analysis. Based on plots of LCC/UCL Hipparcos stars in CMDs and HRDs, we find that ages derived from the HRD are systematically older than CMD and Li ages; the HRD ages are model-dependent whereas CMD and $\mathrm{Li}$ ages are primarily empirically anchored. This difference can explain the discrepancy between our young age and previously claimed older ages of UCL/LCC. Because of the importance of accurate ages in many astrophysical phenomena, a thorough cross-calibration of various age-dating methods for young stars is in urgent need.

This research has made use of the SIMBAD and Vizier databases, operated at CDS, Strasbourg, France. We thank the anonymous referee for useful and constructive comments. This research was partially supported by NASA grants to the University of Georgia and UCLA.

\section{REFERENCES}

Baraffe, I., Chabrier, G., Allard, F., \& Hauschildt, P. H. 1998, A\&A, 337, 403 Chen, C. H., Jura, M., Gordon, K. D., \& Blaylock, M. 2005, ApJ, 623, 493 Currie, T., Kenyon, S. J., Balog, Z., et al. 2008, ApJ, 672, 558 de la Reza, R., Jilinski, E., \& Ortega, V. G. 2006, AJ, 131, 2609 de Zeeuw, P. T., Hoogerwerf, R., de Bruijne, J. H. J., Brown, A. G. A., \& Blaauw, A. 1999, AJ, 117, 354

Fernández, D., Figueras, F., \& Torra, J. 2008, A\&A, 480, 735 Fuhrmeister, B., \& Schmitt, J. H. M. M. 2003, A\&A, 403, 247 Hambly, N. C., MacGillivray, H. T., Read, M. A., et al. 2001, MNRAS, 326, 1279

Høg, E., Fabricius, C., Makarov, V. V., et al. 2000, A\&A, 355, L27

Kenyon, S. J., \& Hartmann, L. 1995, ApJS, 101, 117

Lawson, W. A., \& Crause, L. A. 2005, MNRAS, 357, 1399

Mamajek, E. E., Meyer, M. R., \& Liebert, J. 2002, AJ, 124, 1670

Ortega, V. G., de la Reza, R., Jilinski, E., \& Bazzanella, B. 2002, ApJ, 575, L75

Pecaut, M. J., Mamajek, E. E., \& Bubar, E. J. 2012, ApJ, 746, 154

Perryman, M. A. C., Lindegren, L., Kovalevsky, J., et al. 1997, A\&A, 323, L49

Preibisch, T., Guenther, E., \& Zinnecker, H. 2001, AJ, 121, 1040

Siess, L., Dufour, E., \& Forestini, M. 2000, A\&A, 358, 593

Song, I., Bessell, M. S., \& Zuckerman, B. 2002, ApJ, 581, L43

Song, I., Zuckerman, B., \& Bessell, M. S. 2003, ApJ, 599, 342

Song, I., Zuckerman, B., \& Bessell, M. S. 2004, ApJ, 614, L125

Stauffer, J. R., Hartmann, L. W., Prosser, C. F., et al. 1997, ApJ, 479, 776

van Leeuwen, F. 2007, A\&A, 474, 653

Voges, W., Aschenbach, B., Boller, Th., et al. 1999, A\&A, 349, 389

Voges, W., Aschenbach, B., Boller, T., et al. 2000, IAU Circ., 7432, 1

Zuckerman, B., \& Song, I. 2004, ARA\&A, 42, 685

Zuckerman, B., Song, I., \& Bessell, M. S. 2004, ApJ, 613, L65 